\title{
Barriers to optimal breast milk provision in the neonatal intensive care unit
}

\author{
Meera N. Sankar $\mathbb{D}^{1 凶}$, Ya'el Weiner ${ }^{1,2}$, Neha Chopra ${ }^{1,2}$, Peiyi Kan ${ }^{1,2}$, Zakiyah Williams ${ }^{3}$ and Henry C. Lee (iD) ${ }^{1,2}$ \\ (c) The Author(s), under exclusive licence to Springer Nature America, Inc. 2021
}

OBJECTIVE: This study examines comprehensive patient and process factors that influence breast milk use in the NICU setting. STUDY DESIGN: We examined the association of maternal, neonatal, and family factors and lactation support systems to identify gaps in breast milk use in a retrospective study of 865 infants born in 23-41 weeks gestation admitted to the NICU.

RESULTS: Breast milk at discharge for all infants was $89.3 \%$, for extremely preterm $82.3 \%$, moderately preterm $91.4 \%$, late preterm $86.5 \%$, and term 92.7\%. Prematurity (OR 0.31 [0.17-0.56]), low birth weight, morbidities, Black maternal race (OR 0.20 [0.07-0.57]) and public insurance (OR 0.54 [0.34-0.85]) were associated with decreased breast milk use. Early initiation of feeds was associated with increased breast milk use.

CONCLUSIONS: There is a need to increase social as well as hospital support systems to address gaps in breast milk use in the NICU.

Journal of Perinatology (2022) 42:1076-1082; https://doi.org/10.1038/s41372-021-01275-4

\section{INTRODUCTION}

Preterm infants are a high-risk population, and prematurity is a leading cause of newborn morbidity and mortality in the United States. In the high-risk preterm population, early human milk feeding is associated with a decrease in mortality and morbidity in the Neonatal Intensive Care Unit (NICU), decreased rates of illness and rehospitalization in the first year of life and improved neurodevelopmental outcomes [1-4]. This neonatal population has a profound need for lactation support $[5,6]$.

Studies have shown that breastfeeding rates among late preterm infants born at $34^{0 / 7}-36^{6 / 7}$ weeks gestation are lower than rates among earlier preterm infants. Late preterm infants are at risk for poor breastfeeding due to immature oral feeding skills. Very premature infants are at a higher risk for several morbidities, including necrotizing enterocolitis, and it is thus possible that health care providers are more motivated to ensure early breast milk feeding for these infants [7]. Term infants admitted to the NICU for respiratory distress, neurological, or cardiac disorders lose the initial skin-to-skin contact with their mothers and have delayed establishment of breastfeeding compared with healthy term infants [7-10].

Research has shown that targeted lactation support interventions for extremely low birth weight neonates less than 1000 grams and very low birth weight neonates less than 1500 grams admitted to the NICU have increased the human milk use in this high-risk population [4, 11-18]. However, there is a gap in our knowledge about lactation support systems for late preterm and term neonates with complex conditions admitted to the NICU. Furthermore, the impact of social factors such as family transportation issues and language barriers on lactation outcomes have not been examined at length across gestational age groups of infants admitted to the NICU.
While larger population-based studies have shown demographic and medical risks for lack of breastfeeding success, there is a gap in knowledge in (1) more detailed individual level data both before and after birth (conditions of pregnancy, labor, and delivery, barriers to parental visits to the NICU, skin-to-skin contact), and (2) process level factors, particularly related to lactation consultation timing and frequency for patients in the NICU.

In this present study, we examined the patient and systems barriers to optimal breast milk use in all infants, including preterm, late preterm and term infants admitted to a large, level IV NICU. We also studied the relationship of the availability of lactation services support, as well as maternal demographics, morbidities related to being preterm, family factors, and diagnosis on admission to the NICU that could influence breast milk use at discharge.

\section{METHODS}

We performed a retrospective study of all babies admitted to the Lucile Packard Children's Hospital (LPCH) Stanford NICU during the time period 9/ 2015-6/2018. We chose the study start timeline based on the concerted effort to increase coverage and provide consistent lactation support in the LPCH NICU since September 2015. The study was approved by Stanford University's Institutional Review Board and informed consent was waived due to the nature of research that involved de-identified data analysis.

During this study period, there were a total of 1233 infants admitted to the NICU. We limited our study cohort to infants, who were either inborn or infants who were outborn but transferred to our NICU on the first or second day of age. We included 139 infants who were transferred out to other hospitals for convalescence along with the discharged infants. There were 35 records with missing or unknown data, leaving 865 infants for the final data analysis.

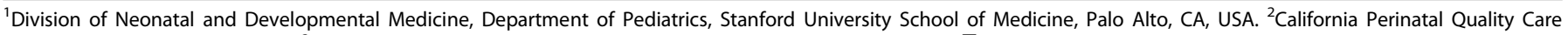
Collaborative, Stanford, CA, USA. ${ }^{3}$ Lactation services, Lucile Packard Children's Hospital, Stanford, CA, USA. ${ }^{凶}$ email: Mnsankar@stanford.edu 
We examined maternal and infant factors for all the admitted infants. Maternal characteristics include demographic factors, number of previous pregnancies, medical complications during pregnancy such as preeclampsia, gestational diabetes, preterm labor, preterm premature rupture of membranes, and use of antenatal steroids, singleton vs. multiple gestation, type of insurance, race/ethnicity, and characteristics of labor and delivery. Neonatal characteristics included inborn versus outborn, infant's gestational age, gender, birth weight, head circumference, Apgar scores, need for resuscitation in the delivery room, characteristics of resuscitation support, diagnosis on admission, neonatal morbidities such as sepsis, bronchopulmonary dysplasia, necrotizing enterocolitis, intraventricular hemorrhage, periventricular leukomalacia, retinopathy of prematurity, and need for nasogastric feeds, gastrostomy tube feeds, need for oxygen support, mortality, length of stay, and weight at discharge.

Maternal ethnicity was categorized as non-Hispanic versus Hispanic, and the race was categorized as White, Black, Asian/Pacific Islander, Native American, or other based on admission demographic data. Insurance was categorized as none, public, or private.

Infant feeding factors included data on infant's first feed, nature of initial feed and infant's feed $24 \mathrm{~h}$ prior to discharge or transfer. For the infant's first feed, we categorized data as receiving breast milk, any breastfeeding, donor milk, or formula. In our institution, although donor milk is available to all newborns admitted to the NICU, it is specifically incorporated in the feeding guidelines for preterm infants less than 32 weeks with parental assent. For infants born at $32^{1 / 7}$ weeks or later, donor milk use is not mandated as part of the feeding guidelines, but available for use. Preterm infants less than 32 weeks are typically transitioned off donor milk at 34 weeks postmenstrual age. For the infant's feeds $24 \mathrm{~h}$ prior to discharge or transfer, we categorized breast milk used as a combination of breast milk and a few bottles of preterm formula (as most preterm babies get discharged on the combination regimen) or breastfeeding (due to the inclusion of term and late preterm babies), and exclusive formula use without any breast milk use or breastfeeding.

Family factors studied included transportation issues, language barriers necessitating interpreter use due to limited English proficiency (LEP), childcare factors, and some combination of these various factors. We abstracted information from the medical social worker's documentation in the infant's chart regarding transportation vouchers provided to families, childcare issues expressed by parents, and the need for interpreter use to communicate with families. LEP was defined as the need for interpreter support as documented in the medical social worker's note in the chart. We defined a combination of family factors as noted in the chart documentation if more than one issue such as LEP, transportation needs, and childcare issues faced by families limiting access to lactation consultant support.

We studied the effects of admission diagnosis to the NICU, such as respiratory distress, hypoglycemia, jaundice, neurological conditions (apnea, seizures), and cardiac disease in term and late preterm infants.

During the study period, we had 1.9 full-time equivalent (FTE) International Board-Certified Lactation Consultants (IBCLCS) dedicated to the NICU. We had consistent daytime coverage between 8 AM to 5 PM and evening coverage on some days until 8 PM. Bedside nursing had not received formal lactation support training and we were not considered as a Baby-Friendly Hospital during the study period.

We collected extensive data on lactation systems variables including the following: date of first IBCLC consult, the timing of first IBCLC consult in relation to infant's birth, frequency of IBCLC support in the first week, frequency of IBCLC support during NICU stay, and IBCLC support prior to discharge. We defined the date of the first lactation support as the first $I B C L C$ visit of the mother. For the frequency of lactation support in the first week, we defined the number of IBCLC visits the mother received during that time period. Due to varied lengths of stay for late preterm versus extremely preterm infants admitted to the NICU, we defined the frequency of lactation support during NICU stay as the average number of IBCLC visits per week of infant's hospitalization in the NICU. Lactation support prior to discharge was defined as a binary variable depending on whether the mother received an IBCLC visit in the last week prior to her infant's discharge from the NICU.

\section{Statistical analysis}

Our primary outcome was the use of breast milk prior to discharge. We defined the mother's own milk at discharge and not donor milk as the primary outcome for this study. We performed bivariate analysis to examine relationships between predictors and outcomes. In addition to the entire population, we also stratified the infants into four subgroups: extremely preterm ( $\leq 30$ weeks), moderate preterm $\left(30^{1 / 7}-33^{6 / 7}\right.$ weeks), late preterm (34 $1 / 7-36^{6 / 7}$ weeks), and term ( 37 weeks and greater) and performed analysis in the various subgroups. Chi-square test and Student's $t$-test were used for categorical and continuous variables, respectively.

We built multivariable logistic regression models to identify factors associated with no breast milk use at discharge. We adjusted for the infant's gestational age, gender, maternal race/ethnicity, maternal age, multiple gestations, length of stay at discharge, and insurance factors. Adjusted odds ratios (ORs) were calculated with $95 \%$ confidence intervals (Cls) for the association of maternal and sociodemographic factors, factors related to labor and delivery, infant factors, and lactation support factors with no breast milk use prior to discharge. We performed further analyses to study the impact of maternal race/ethnicity, language barriers seen in non-English speaking mothers, and family transportation issues on the lactation variables. An alpha level of $P<0.05$ was used as the cutoff for statistical significance. Statistical analyses were computed using SAS 9.1 (SAS, Cary, North Carolina).

\section{RESULTS}

Breast milk use at discharge for the total population was $89.4 \%$. Although any breast milk use at discharge in all NICU admits was high at $89.4 \%$, we found that the rates were significantly less for certain subgroups: $80 \%$ in the extremely preterm (OR, $0.31 ; 95 \%$ $\mathrm{Cl}, 0.17-0.56)$ and $86 \%$ in the late preterm $(\mathrm{OR}, 0.48 ; 95 \% \mathrm{Cl}$, 0.24-0.97) infants compared to the overall NICU population.

Maternal characteristics, neonatal factors, infant feeding/lactation factors, and breast milk use versus no breast milk use at discharge are presented in Tables 1, 2, and 3, respectively. The gestational age breakdown for breast milk use at discharge is as follows: extremely preterm infants $80.4 \%$, moderate preterm $91.3 \%$, late preterm $86.5 \%$ and term $93 \%$.

The joint effects of maternal, neonatal, infant feeding and lactation support factors on no breast milk feeds at discharge in all infants and for the various subgroups of extremely preterm, term, and late preterm infants is shown in Table 4. The significant effects of infant's gestational age, race, neonatal morbidities, public insurance, lack of lactation support at discharge, lack of direct breastfeeding in the NICU on no breast milk use at discharge persisted after multivariable logistic regression.

Morbidities such as bronchopulmonary dysplasia, necrotizing enterocolitis, and use of donor milk were associated with significantly decreased breast milk use in extremely preterm infants. Initial feeding with formula was associated with significantly decreased breast milk use in term and late preterm infants. We found that hypoglycemia and neurological diagnosis on admission (documented as apnea), and nasogastric feeds at discharge was associated with significantly decreased breast milk use at discharge in term infants. Respiratory distress in the newborn was not associated with any difference in breast milk use between the groups in term and late preterm infants.

In examining associations of race/ethnic categories, we found that Black (OR 0.30; 95\% Cl, 0.10-0.93) and Native American (OR $0.21 ; 95 \% \mathrm{Cl}, 0.05-0.87)$ maternal race had lower adjusted odds of breast milk used at discharge compared to other races. However, we did not find a difference between Hispanic and non-Hispanic ethnic groups regarding the primary outcome of breast milk use at discharge.

In assessing the barriers to care for NICU families, we found infants of families with transportation issues (OR $0.53 ; 95 \% \mathrm{Cl}$, $0.31-0.93$ ) and those on public insurance (OR $0.48 ; 95 \% \mathrm{Cl}$, $0.29-0.77$ ) had significantly less breast milk use at discharge. LEP families with a need for interpreter support did not have a significant decrease in breast milk use at discharge.

We found that IBCLC support at discharge and the opportunity for mothers to practice direct breastfeeding during NICU stay was associated with increased breast milk use at discharge for all infants admitted to the NICU. Lack of IBCLC support prior to 
Table 1. Maternal characteristics of all infants admitted to the NICU.

\begin{tabular}{|c|c|c|c|}
\hline $\begin{array}{l}\text { Maternal } \\
\text { characteristics }\end{array}$ & $\begin{array}{l}\text { Breast milk } \\
\text { use at } \\
\text { discharge } \\
N(\%)\end{array}$ & $\begin{array}{l}\text { No breast milk } \\
\text { use at } \\
\text { discharge } \\
N(\%)\end{array}$ & $P$ value \\
\hline Sample size & $777(89.83)$ & $88(10.2)$ & \\
\hline Maternal age (years) & & & 0.69 \\
\hline$<20$ & $26(89.7)$ & $3(10.3)$ & \\
\hline $20-29$ & $218(87.9)$ & $30(12.1)$ & \\
\hline $30-39$ & $415(90.0)$ & $46(10.0)$ & \\
\hline$\geq 40$ & $82(92.1)$ & $7(7.9)$ & \\
\hline Maternal race & & & $0.01^{*}$ \\
\hline White & $281(90.6)$ & $29(9.4)$ & ref \\
\hline Black & $16(72.7)$ & $6(27.3)$ & $0.01^{*}$ \\
\hline Asian/Pacific Islander & $179(91.8)$ & $16(8.2)$ & 0.66 \\
\hline Native American & $6(66.7)$ & $3(33.7)$ & $0.03^{*}$ \\
\hline Other & $264(88.9)$ & $33(11.1)$ & 0.48 \\
\hline \multicolumn{4}{|l|}{ Maternal ethnicity } \\
\hline Non-Hispanic & $428(89.7)$ & $49(10.3)$ & ref \\
\hline Hispanic & 317 (89.5) & $37(10.5)$ & 0.93 \\
\hline Primigravida & $222(90.6)$ & $23(9.4)$ & ref \\
\hline Multigravida & $380(91.1)$ & $37(8.9)$ & 0.82 \\
\hline Multiple births & $97(89.8)$ & $11(10.2)$ & 0.97 \\
\hline Preeclampsia & $74(91.4)$ & 7 (8.6) & 0.43 \\
\hline Gestational diabetes & $153(90.0)$ & $17(10.0)$ & 0.93 \\
\hline Preterm labor & $152(88.9)$ & $19(11.1)$ & 0.81 \\
\hline $\begin{array}{l}\text { Premature rupture of } \\
\text { membranes }\end{array}$ & 127 (87.6) & $18(12.4)$ & 0.81 \\
\hline Antenatal steroids & $331(87.8)$ & $46(12.2)$ & 0.06 \\
\hline Chorioamnionitis & $56(90.3)$ & $6(9.7)$ & 0.64 \\
\hline \multicolumn{4}{|l|}{ Health insurance } \\
\hline Public & $347(86.8)$ & $53(13.3)$ & $<0.01^{* *}$ \\
\hline Private & 391 (92.4) & $32(7.6)$ & ref \\
\hline $\begin{array}{l}\text { Limited English } \\
\text { Proficiency }\end{array}$ & $147(92.5)$ & $12(7.5)$ & 0.23 \\
\hline Transportation issues & $149(85.6)$ & $25(14.4)$ & $0.04^{*}$ \\
\hline Outborn & $214(86.6)$ & $33(13.4)$ & 0.06 \\
\hline Gestational age (weeks) & & & $<0.001^{* * *}$ \\
\hline$<30$ & $126(82.4)$ & $27(17.6)$ & $<0.01^{* *}$ \\
\hline $30-33$ & $149(91.4)$ & $14(8.6)$ & 0.57 \\
\hline $34-36$ & $103(86.6)$ & $16(13.4)$ & $0.03^{*}$ \\
\hline $37-41$ & $399(92.8)$ & $31(7.2)$ & ref \\
\hline \multicolumn{4}{|l|}{ Gender } \\
\hline Male & $461(89.7)$ & $53(10.3)$ & ref \\
\hline Female & $316(90.0)$ & $35(10.0)$ & 0.87 \\
\hline \multicolumn{4}{|l|}{ Birth weight (grams) } \\
\hline $400-750$ & $34(72.3)$ & $13(27.7)$ & 0.10 \\
\hline $751-1000$ & $53(93.0)$ & $4(7.0)$ & 0.10 \\
\hline $1001-1500$ & $103(83.7)$ & $20(16.3)$ & ref \\
\hline $1501-2000$ & $80(96.4)$ & $3(3.6)$ & $<0.01^{* *}$ \\
\hline $2001-2500$ & $66(90.4)$ & $7(9.6)$ & 0.19 \\
\hline$>2501$ & 405 (91.4) & $38(8.6)$ & $0.01^{*}$ \\
\hline
\end{tabular}

discharge (OR $0.27 ; 95 \% \mathrm{Cl}, 0.15-0.51)$ was significantly associated with no breast milk use in all infants. We found that the average number of IBCLC visits per week of hospitalization (OR 4.43; $95 \%$ $\mathrm{Cl}$ 1.81-10.8) was significantly associated with increased breast milk use at discharge in all infants specifically in the subgroup of extremely preterm infants.

We examined the effects of race/ethnicity on the IBCLC support and barriers to care encountered by families (Table 5). We found that although there were no differences in the frequency of IBCLC support in the first week and during NICU stay, Hispanic and nonHispanic Black mothers received less IBCLC support prior to discharge $(35 \%, P$ value $=0.008)$ compared to non-Hispanic White (46\%) and Asian (49\%) mothers. Hispanic patients also had significant barriers to care $(P$ value $<0.001)$ such as transportation issues, need for interpreter support (36\%) compared to nonHispanic White (11\%) and Asian (16\%) mothers.

\section{DISCUSSION}

We found that use of any breast milk prior to discharge was high at $89 \%$ (with a significant decrease in subgroups of extremely preterm $(80 \%)$ and late preterm (86\%)) in our cohort of infants admitted to a large regional NICU over a 2-year period. In a NICU with relatively high rates of breast milk use, we found inequities in outcomes and process measures. Donor milk use in extremely preterm infants and use of formula in late preterm and term infants was associated with significantly decreased breast milk use. Black and Native American race, families with public insurance and transportation issues were associated with significantly decreased breast milk use at discharge. Lactation support systems contributed to a significant increase in breast milk use in all infants.

Previous studies have shown varying breast milk feeding rates of $28-60 \%$ at discharge for VLBW infants [7, 9, 10, 19]. We believe that our rates are higher than previous studies due to the inclusion of all (preterm and term) babies admitted to the NICU, recent emphases on the use of breast milk and associated quality improvement efforts. There is currently a paucity of reports in the literature on accurate breast milk use at discharge on late preterm and term babies admitted to the NICU. We believe our study sheds light on this important subgroup of infants admitted to the NICU.

Research has shown that targeted lactation support interventions for neonates admitted to the NICU have increased human milk use in the high-risk NICU population [11-15, 17, 18, 20]. Similar to a study by Gharib et al. in 2017 [4], we found that the provision of full-time dedicated lactation support is associated with an increase in breastfeeding in both preterm and late preterm infants. There was a concerted effort to increase IBCLC coverage in our NICU since September 2015. We found that the age at first IBCLC visit or hours to first IBCLC visit did not make a difference in the primary outcome. The frequency of IBCLC support throughout the NICU stay made a significant difference in increasing breast milk use in extremely preterm, late preterm, and term infants. Although there was an association between the lack of IBCLC support prior to discharge and no breast milk use, it is likely that this finding is in many cases is due to mothers who had stopped breastfeeding prior to discharge, and therefore the IBCLC's did not visit them. It would be appropriate for IBCLC's not to visit mothers in the NICU after cessation of milk production.

We found that mothers of Hispanic, non-Hispanic Black, and Native American infants received fewer IBCLC visits in the last week prior to discharge compared to non-Hispanic White and non-Hispanic Asian infants. In contrast to these findings, we did not find a difference either in the frequency of IBCLC visit during the first crucial week or the number of IBCLC visits per week of NICU hospitalization among the various race/ethnic groups. Although Black and Native American mothers were a small part 
Table 2. Neonatal characteristics of all infants admitted to the NICU.

\begin{tabular}{|c|c|c|c|}
\hline Neonatal characteristics & Breast milk use at discharge $N(\%)$ & $\begin{array}{l}\text { No breast milk use at discharge } \\
N(\%)\end{array}$ & $P$ value \\
\hline Mode of delivery & & & 0.69 \\
\hline Cesarean & $443(89.1)$ & $54(10.9)$ & 0.43 \\
\hline \multicolumn{4}{|l|}{ Resuscitation } \\
\hline \multicolumn{4}{|l|}{ Morbidities } \\
\hline Sepsis & $31(75.6)$ & $10(24.4)$ & $0.002^{* *}$ \\
\hline Chronic lung disease & $4(33.3)$ & $8(66.7)$ & $<0.001^{* * *}$ \\
\hline Necrotizing enterocolitis & $9(56.3)$ & $7(43.8)$ & $<0.001^{* * * *}$ \\
\hline Retinopathy of prematurity & $61(78.2)$ & $17(21.8)$ & $0.002^{* *}$ \\
\hline Death & $28(93.3)$ & $2(6.7)$ & 0.52 \\
\hline Length of stay (in days) & $32.2 \pm 34.1$ & $70.6 \pm 57.8$ & $<0.0001^{*}$ \\
\hline \multicolumn{4}{|l|}{ Diagnosis on admission } \\
\hline Respiratory distress & $244(90.7)$ & $25(9.3)$ & 0.57 \\
\hline Apnea & $4(50)$ & $4(50)$ & $<0.01^{* *}$ \\
\hline
\end{tabular}

${ }^{*} p<0.05,{ }^{* *} p<0.01$, and ${ }^{* *} p<0.001$.

Table 3. Infant feeding and lactation factors for all infants admitted to the NICU.

\begin{tabular}{|c|c|c|c|}
\hline Infant feeding and lactation factors & Breast milk use at discharge $N(\%)$ & No breast milk use at discharge $N(\%)$ & $P$ value \\
\hline \multicolumn{4}{|l|}{ Infant's initial feed } \\
\hline Breast milk & $461(91.8)$ & $41(8.2)$ & ref \\
\hline Breastfeeding & $31(96.9)$ & $1(3.1)$ & 0.32 \\
\hline Donor milk & $59(73.8)$ & $21(26.3)$ & $<0.01^{*}$ \\
\hline Formula & $16(59.3)$ & $11(40.7)$ & $<0.01^{*}$ \\
\hline Any combination & $125(96.2)$ & $5(3.8)$ & 0.10 \\
\hline Hours to first IBCLC visit (hours) & $78.5 \pm 249.6$ & $60.5 \pm 94.3$ & 0.57 \\
\hline Number of IBCLC visits during the first week & $3.0 \pm 1.8$ & $2.6 \pm 2.1$ & 0.07 \\
\hline Number of IBCLC visits per week of hospitalization & $2.0 \pm 1.8$ & $0.8 \pm 1.0$ & $<0.0001^{* * *}$ \\
\hline \multicolumn{4}{|l|}{ IBCLC support at discharge } \\
\hline Yes & $330(96.5)$ & $12(3.5)$ & $<0.01^{*}$ \\
\hline
\end{tabular}

${ }^{*} p<0.01,{ }^{* *} p<0.0001$, and IBCLC International Board-Certified Lactation Consultant.

our NICU population, they had good support with IBCLC visits during their infant's NICU stay, especially during the initial weeks of hospitalization. It is possible that parents with less flexible work schedules are unable to take time off from work for a long period of time to take advantage of the IBCLC support in the NICU. Although our IBCLCs use interpreters to communicate with nonEnglish speaking families, language barriers to explicitly communicate a request such as the need for lactation support to a NICU team member during visits to the NICU could be contributing to the discrepancy in lactation support. The IBCLC support may have become inconsistent over time, and we do see that as a gap in lactation support that needs to be addressed to improve and achieve equity in lactation support to diverse NICU families. We speculate that fewer IBCLC visits at discharge could also be related to factors such as cessation of breast milk production by Hispanic mothers prior to discharge compared to White mothers as shown in studies by Parker et al. [19, 21-23] We acknowledge that due to lack of comprehensive data on skin-to-skin care and hour to first milk expression, we are unable to evaluate the significance of 


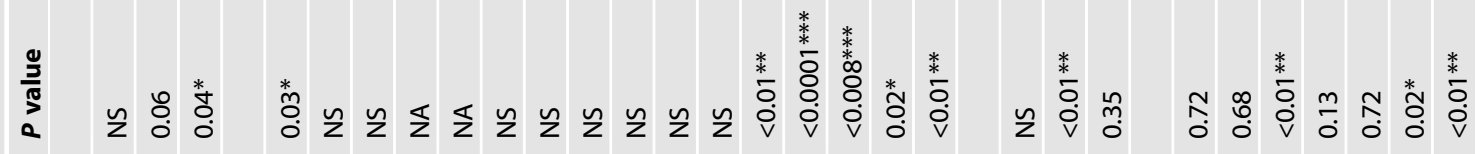

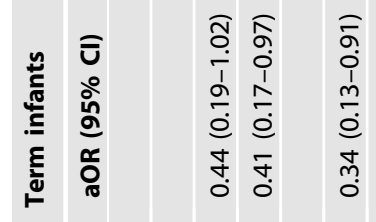

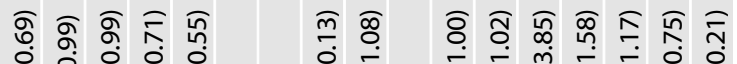

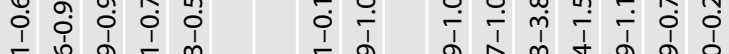
స d. సิ ลิ

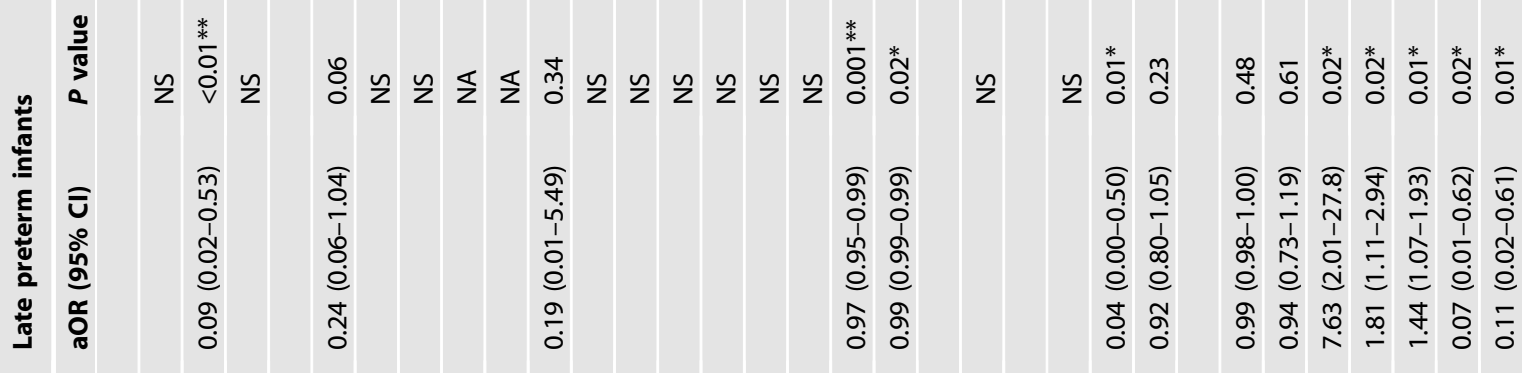

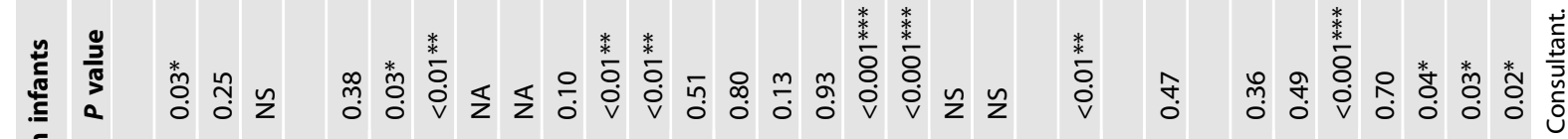

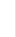
产

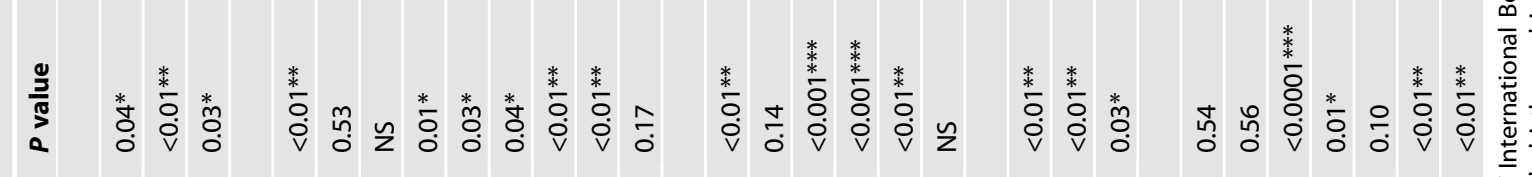

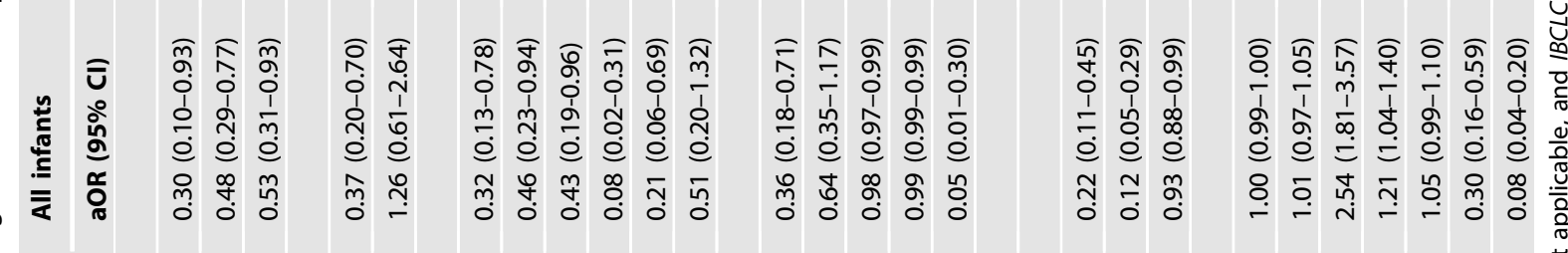

$$
\text { (a) }
$$


Table 5. Association of maternal race/ethnicity with lactation support factors and barriers to care in all infants admitted to the NICU.

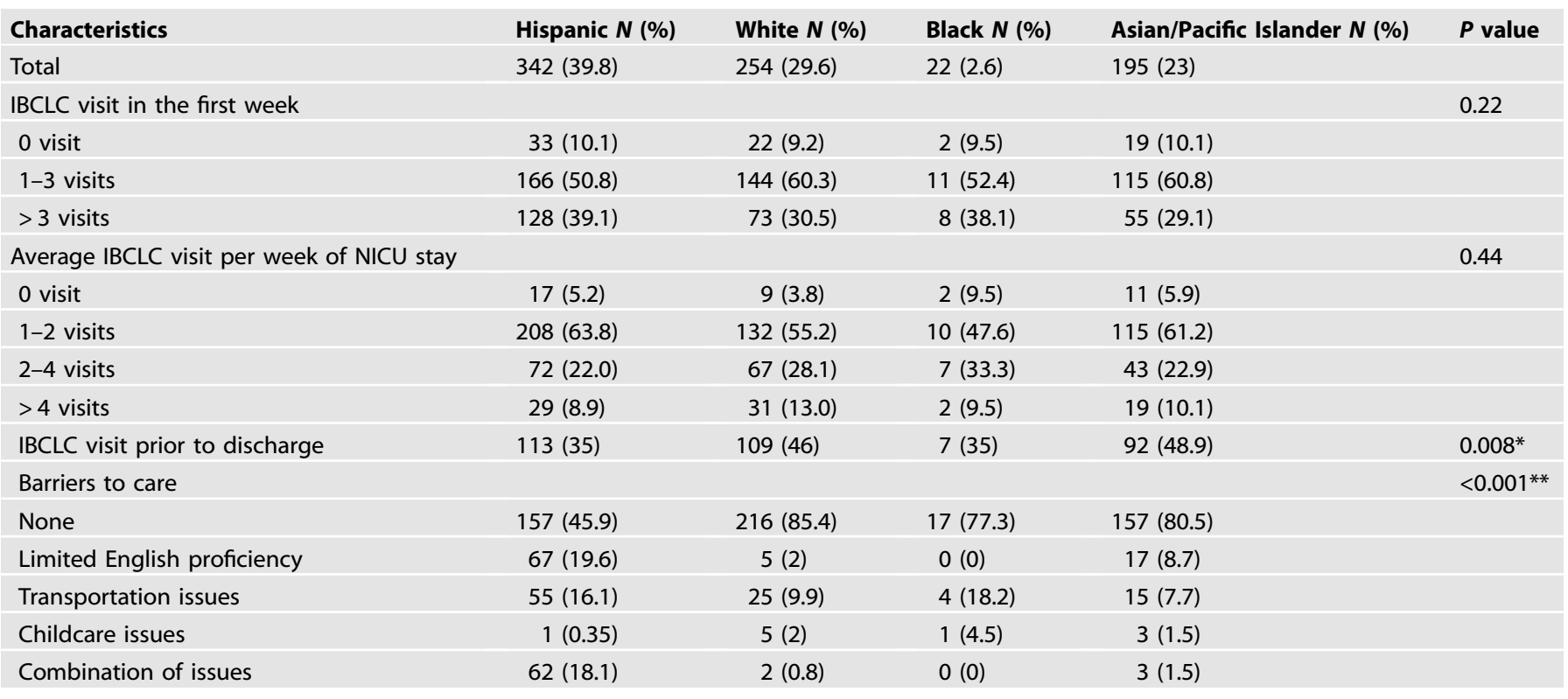

${ }^{*} p<0.01,{ }^{* *} p<0.001$, and $I B C L C$ International Board-Certified Lactation Consultant.

these important variables on increasing breast milk use as shown in previous studies [19].

Recent studies have focused on the association between maternal breastfeeding goals and neighborhood structural factors in increasing breast milk use at discharge [24-26]. The interaction between access to a car and race/ethnicity significantly differed between Black and White mothers, although the human milk feeds at discharge was not significantly affected by access to a car by any racial/ethnic group [25]. In our study, the effects of socioeconomic factors such as public insurance caused a decrease in breast milk use in all infants. We studied family barriers to care that would limit their ability to care for their babies in the NICU such as transportation issues, need for language interpreter use, and childcare issues. We found significant disparities in barriers to care, especially transportation issues limiting NICU visitation among families of Hispanic versus non-Hispanic infants. These family factors could create gaps in receiving adequate lactation support to achieve success in providing breast milk to their infants. Recent equity-focused quality improvement (EF-QI) initiatives that are action-oriented and centered around equity will hopefully help to effectively combat disparities. The EF-QI initiatives integrate equity throughout the fabric of the project and are inclusive, collaborative efforts that prioritize and address the needs of disadvantaged populations [27-29].

Similar to previous studies, opportunities for mothers to practice direct breastfeeding in the NICU were associated with increased breast milk use [11, 16, 19, 23, 30]. Interestingly, we found that in contrast to previous studies, the use of donor milk caused a decrease in breast milk use at discharge. The reason for this finding is unclear. We speculate that increased availability and use of donor milk could potentially result in a delay in establishing an optimal breast milk supply. Since direct breastfeeding in the NICU is shown to have significant effects, efforts should be targeted to ensure mothers visit their infants, especially extremely preterm infants during the weeks prior to discharge to practice and get support from the nursing and lactation team on improving their breastfeeding skills.

Based on limited data on diagnosis categories upon admission to the NICU, we found that "apnea" diagnosis was associated with significantly decreased breast milk use in term infants. As NICUs specialize in treating term infants with surgical disorders, complex congenital heart disease, or neurological disorders such as hypoxic-ischemic encephalopathy receiving hypothermia treatment, obtaining comprehensive data on these infants will be beneficial. We acknowledge our findings are based on data obtained from a single-center, retrospective study. Future studies should focus on breast milk at discharge for a broader population of term, high-risk infants with surgical, neurological, and congenital heart disease admitted to the NICU.

Strengths of our study include the comprehensive data on patient and process-related factors influencing breast milk use in all infants admitted to the NICU. The population studied reflects a racially and ethnically diverse group of babies from a large, regional NICU in California. In contrast to other studies, we obtained data that showed benefits seen in late preterm and term infants in addition to the extremely preterm infant population. We obtained detailed lactation consultant related process factors for the entire duration starting with the baby's admission to the NICU until discharge. Ours is one of the few studies that collected data on family transportation issues, need for interpreter use, and childcare issues that impact breast milk use. We also assessed the differences in lactation process support and barriers to care experienced by families of several racial/ethnic groups. Since the family factors were abstracted from the social worker's note, it is likely that other social issues occurred that were not assessed and documented. We acknowledge this factor as a study limitation. The findings from this study are based on retrospective data collected from a single-center, which is another limitation. Although our population represents a diverse setting in a large California NICU, the results obtained here may not be applicable in other areas of this country and internationally. There was a lack of comprehensive data on important metrics such as hour of first milk expression after birth, skin-to-skin care in the NICU, and breastfeeding before first bottle feeding and so we were unable to assess their effectiveness in our population. This study data was collected prior to the COVID-19 pandemic, and so we are unable to ascertain the various effects such as family visitation policy changes in addition to the disparities faced by low income and minority families due to actual disease burden on the primary outcome of any breast milk use in the NICU. 
In conclusion, our study findings from a diverse population of infants from a large, regional NICU in California showed an overall high rate of breast milk used at discharge in all infants. Extremely preterm infants with morbidities showed decreased breast milk use. Racial/ethnic disparities were noted in breast milk use at discharge. Dedicated NICU Lactation support systems significantly improved breast milk used for all infants. We hope that findings from this study provide a foundation to develop successful targeted interventions to improve outcomes of high-risk infants.

\section{REFERENCES}

1. Lucas A, Cole TJ. Breast milk and neonatal necrotizing enterocolitis. Lancet. 1990;336:1519-23.

2. Meinzen-Derr J, Poindexter BB, Wrage L, Morrow AL, Stoll B, Donovan EF. Role of human milk in extremely low birth weight infants' risk of necrotizing enterocolitis or death. J Perinatol. 2009;29:57-62.

3. Vohr BR, Poindexter BB, Dusick AM, McKinley LT, Wright LL, Langer JC, et al. Beneficial effects of breast milk in the neonatal intensive care unit on the developmental outcome of extremely low birth weight infants at 18 months of age. Pediatrics 2006;118:e115-e123.

4. Gharib S, Fletcher M, Tucker R, Vohr B, Lechner BE. Effect of dedicated lactation support services on breastfeeding outcomes in extremely-low-birth weight neonates. J Hum Lact. 2018;34:728-36.

5. Alves E, Magano R, Amorim M, Nogueira C, Silva S. Factors influencing parent 'reports of facilitators and barriers to human milk supply in neonatal intensive care units. J Hum Lactation. 2016;32:695-703.

6. Greene MM, Rossman B, Patra K, Kratovil AL, Janes JE, Meier PP. Depression, anxiety, and perinatal-specific posttraumatic distress in mothers of very low birth weight infants in the neonatal intensive care unit. J Developmental Behav Pediatrics. 2015;36:362-70.

7. Kair LR, Colaizy TT. Breastfeeding continuation among late preterm infants: barriers, facilitators, and any association with NICU admission? Hosp Pediatr. 2016;6:261-8.

8. Oras $P$, Thernstrom Blomqvist $Y$, Hedberg Nyqvist $K$, Gradin $M$, Rubertsson $C$, Hellstrom-Westas L, et al. Skin-to-skin contact is associated with breastfeeding attainment in preterm infants. Acta Paediatr. 2016;105:783-9.

9. Baley J. \& Committee of Fetus and Newborn. Skin-to-skin care for term and preterm infants in the neonatal ICU. Pediatrics 2015;136:596-9.

10. Kachoria R, Oza-Frank R. Trends in breastfeeding initiation in the NICU by gestational age in Ohio, 2006-2012. Birth 2015;42:56-61.

11. Pineda RG, Foss J, Richards L, Pane CA. Breastfeeding changes for VLBW infants in the NICU following staff education. Neonatal Netw. 2009;28:311-9.

12. Murphy L, Warner DD, Parks J, Whitt J, Peter-Wohl S. A quality improvement project to improve the rate of early breast milk expression in mothers of preterm infants. J Hum Lact. 2014;30:398-401.

13. Castrucci BC, Hoover KL, Lim S, Maus KC. Availability of lactation counseling services influences breastfeeding among infants admitted to neonatal intensive care units. Am J Health Promotion. 2007;21:410-5.

14. Casavant SG, McGrath JM, Burke G, Briere CE. Caregiving factors affecting breastfeeding duration within a neonatal intensive care unit. Adv Neonatal Care. 2015;15:421-8

15. Herich LC, Cuttini M, Croci I, Franco F, Di Lallo D, Baronciani, Zeitlin J. Maternal education is associated with disparities in breastfeeding at time of discharge but not at initiation of enteral feeding in the neonatal intensive care unit. J Pediatrics. 2017;182:59-65. e7.

16. Lee HC, Kurtin PS, Wight NE, Chance K, Cucinotta-Fobes T, Hanson-Timpson TA, et al. A quality improvement project to increase breast milk use in very low birth weight infants. Pediatrics 2012;130:e1679-87.

17. Sisk PM, Lovelady CA, Dillard RG, Gruber KJ. Lactation counseling for mothers of very low birth weight infants: effect on maternal anxiety and infant intake of human milk. Pediatrics 2006;117:e67-e75.

18. Patel S, Patel S. The effectiveness of lactation consultants and counselors on breastfeeding outcomes. J Hum Lact. 2016;32:530-41.

19. Parker MG, Gupta M, Melvin P, Burnham LA, Lopera AM, Moses JM, et al. Racial and ethnic disparities in the use of mother's milk feeding for very low birth weight infants in massachusetts. J Pediatr. 2019;204:134-41.
20. Bixby C, Baker-Fox C, Deming C, Dhar V, Steele C. A multidisciplinary quality improvement approach increases breastmilk availability at discharge from the neonatal intensive care unit for the very-low-birth-weight infant. Breastfeed Med. 2016;11:75-79.

21. Profit J, Gould JB, Bennett M, Goldstein BA, Draper D, Phibbs CS, et al. Racial/ Ethnic Disparity in NICU Quality of Care Delivery. Pediatrics 2017;140:e20170918.

22. Sigurdson K, Mitchell B, Liu J, Morton C, Gould JB, Lee HC, et al. Racial/ethnic disparities in neonatal intensive care: a systematic review. Pediatrics 2019;144: e20183114.

23. Lee HC, Gould JB. Factors influencing breast milk versus formula feeding at discharge for very low birth weight infants in California. J Pediatr. 2009;155:657-62.

24. Fleurant E, Schoeny M, Hoban R, Asiodu IV, Riley B, Meier PP, et al. Barriers to human milk feeding at discharge of very-low-birth-weight infants: maternal goal setting as a key social factor. Breastfeed Med. 2017;12:20-27.

25. Riley B, Schoeny M, Rogers L, Asiodu IV, Bigger HR, Meier PP, et al. Barriers to human milk feeding at discharge of very low-birthweight infants: evaluation of neighborhood structural factors. Breastfeed Med 2016;11:335-42.

26. Vohr BR, Poindexter BB, Dusick AM, McKinley LT, Wright LL, Langer JC, et al. NICHD neonatal research network. Beneficial effects of breast milk in the neonatal intensive care unit on the developmental outcome of extremely low birth weight infants at 18 months of age. Pediatrics 2006;118:e115-23.

27. Reichman V, Brachio SS, Chinonyerem RM, Montoya-Williams D, Peña MM. Using rising tides to lift all boats: equity-focused quality improvement as a tool to reduce neonatal health disparities. Semin Fetal Neonatal Med. 2021:101198.

28. Mercado K, Vittner D, McGrath J. What is the impact of NICU-dedicated lactation consultants? An evidence-based practice brief. Adv Neonatal Care. 2019;19:383-93.

29. Mannel R, Mannel S. Staffing for hospital lactation programs: recommendations from a tertiary care teaching hospital. J Hum Lact. 2006;22:409-17.

30. Brownell EA, Lussier MM, Hagadorn Jl, McGrath JM, Marinelli KA, Herson VC. Independent predictors of human milk receipt at neonatal intensive care unit discharge. Am J Perinatol. 2014;31:891-8.

\section{AUTHOR CONTRIBUTIONS}

MNS and HCL conceptualized and designed the study. MNS drafted the initial version of the manuscript, and MNS and $\mathrm{HCL}$ critically reviewed and revised the manuscript. YW, NC, and ZW collected data and critically reviewed the manuscript. PK performed the data analysis and critically reviewed the manuscript.

\section{FUNDING}

This work was supported by grant support from the Maternal and Child Health Research Institute, MCHRI, Stanford University. Award PTA 1177593-118-JHAGI to MNS.

\section{COMPETING INTERESTS}

The other authors declare no conflicts of interest relevant to this article. Dr. Lee is a paid consultant to Lansinoh Laboratories, Inc, and received research grant funding from Mead Johnson Nutrition.

\section{ADDITIONAL INFORMATION}

Correspondence and requests for materials should be addressed to Meera N. Sankar.

Reprints and permission information is available at http://www.nature.com/ reprints

Publisher's note Springer Nature remains neutral with regard to jurisdictional claims in published maps and institutional affiliations. 